\title{
The Comparison of Total Phenolics, Total Antioxidant, and Anti-Tyrosinase Activities of Korean Sargassum Species
}

\author{
Su Hyeon Baek, ${ }^{1}$ Lei Cao, ${ }^{1}$ Seung Jin Jeong, ${ }^{2}$ Hyeung-Rak Kim, ${ }^{1,2}$ Taek Jeung Nam, \\ and Sang Gil Lee $\mathbb{D}^{1}$ \\ ${ }^{1}$ Department of Food Science and Nutrition, Pukyong National University, 45 Yongso-Ro, \\ Nam-Gu Busan 48513, Republic of Korea \\ ${ }^{2}$ Department of Smart Green Technology Engineering, Pukyong National University, 45 Yongso-Ro, \\ Nam-Gu Busan 48513, Republic of Korea \\ ${ }^{3}$ The Future Fishers Food Research Center, Institute of Fisheries Sciences, Pukyong National University, \\ 45 Yongso-Ro, Nam-Gu Busan 48513, Republic of Korea
}

Correspondence should be addressed to Sang Gil Lee; sglee1125@pknu.ac.kr

Received 4 November 2020; Revised 21 December 2020; Accepted 6 January 2021; Published 18 January 2021

Academic Editor: Muhammad H. Alu'datt

Copyright ( 2021 Su Hyeon Baek et al. This is an open access article distributed under the Creative Commons Attribution License, which permits unrestricted use, distribution, and reproduction in any medium, provided the original work is properly cited.

Sargassum species, a group of marine brown algae consumed in Asian countries, have shown various health benefits, such as improving the conditions of cardiovascular disease, osteoarthritis, and hypopigmentation. Also, these benefits are associated with their phenolic content and strong antioxidant capacities. However, the antioxidant capacities of different Sargassum species had not been thoroughly explored and compared. Thus, this study aimed to compare the total phenolic contents, total flavonoid contents, total antioxidant capacities, and anti-tyrosine activity of eleven Sargassum species harvested off the Korean coast. The results revealed that the total phenolic content (from 20.57 to $88.97 \mathrm{mg}$ gallic acid equivalent/g dry weight (dw)), flavonoid content (from 22.08 to $82.33 \mathrm{mg}$ quercetin equivalent/g dw), anti-tyrosinase activity (from 13.30 to $126.30 \mathrm{mg}$ kojic acid equivalent/dw), and antioxidant capacities of the 11 Sargassum species had wide ranges. Among them, S. miyabei Yendo and S. hemiphyllum showed the highest total antioxidant capacities while S. miyabei Yendo exhibiting the highest total phenolic and flavonoid contents. The highest anti-tyrosinase activity was seen in S. fillicinum and S. yendoi. Sargahydroqunoic acid and sargachromanol, two alga-derived meroterpenoid compounds with strong antioxidant activity, were detected and quantified in S. miyabei Yendo and S. serratifolium. Our findings guarantee further investigation of the health benefits of Sargassum species and maximize the commercial usage of these species.

\section{Introduction}

Seaweed farming cultivates and harvests seaweed for a food source and non-food usage. According to the World Fisheries and Aquaculture, global production of farmed seaweeds increased from 13.5 million tons in 1995 to over 30 million tons in 2016. Sargassum, family Sargassaceae, order Fucales Kylin, class Cyclosporophyceae, is one of the eight genera that provide the majority $(96.8 \%)$ of the world seaweed aquaculture production in 2018 [1]. With 361 taxonomically accepted species, Sargassum is considered one of the richest genera among the large brown seaweed [2].
Sargassum species inhabit shallow water and coral reefs throughout the world. Asian countries have a long history of consuming Sargassum species. The nutritional assessment showed that Sargassum contains a diversity of bioactive compounds, including vitamin $C$, vitamin $E, \beta$-carotene and dietary fiber, phenolic compounds, and polysaccharides. The known health benefits of Sargassum species include reducing the risk of chronic diseases including obesity, types of cancers, type 2 diabetes, and neurodegeneration diseases [3].

Endogenous reactive oxygen species (ROS) have critical physiological functions, such as signal transmission and defense against external substances. However, excessive 
generation of ROS can cause oxidative stress which contributes to the development of various chronic diseases, such as type 2 diabetes and cardiovascular disease [4]. Along with antioxidant enzymes, dietary antioxidant intake is essential for the physiological redox balance [5]. Compared to the widely studied fruits and vegetables-derived antioxidants, algae are less acknowledged as a potent antioxidant source. A growing body of evidence shows that algae can synthesize a great variety of primary and secondary metabolites with strong antioxidant capacity, including carotenoids, phenolic compounds, and vitamins [6].

Tyrosinase is a multi-copper enzyme that functions as a rate-limiting enzyme in the synthesis of melanin, critical for undesired browning of fruits and vegetables and skin pigmentation [7]. Screening and development of effective tyrosinase inhibitors are of great importance for their applications in the cosmetic and food industries.

To the best of our knowledge, the nutritional facts of different Sargassum species had not been thoroughly compared. Large quantities of different species of Sargassum grow along the shores of South Korea. Here, we analyzed eleven species collected off the Korean coast by the Marine Bio-Resource Information System (MBRIS) in 2018, including S. hemiphyllum, S. coreanum, S. horneri, S. confusum, S. siliquastrum, S. miyabei Yendo, S. serratifolium, S. filicinum, S. micracanthum, S. macrocarpum, and S. yendoi. By analyzing and comparing their antioxidant ability, phenolic and flavonoid contents, and anti-tyrosinase activities, we want to provide information for their use in human and animal nutrition. By measuring the total phenolic content, total flavonoid content, antioxidant capacities, and anti-tyrosinase activity of 11 species of Sargassum inhabiting in Korean coast, this study tried to present a nutrition spectrum of Korean Sargassum species and provide information for optimizing Sargassum culture based on their nutrition density.

\section{Materials and Methods}

2.1. Sample Preparation. Eleven standardized Sargassum extract powders (50 g of dried Sargassum sample was extracted with 10 volumes of $70 \%$ ethanol) were officially obtained from MBRIS. For analysis, $10 \mathrm{mg}$ of Sargassum extract powders were dissolved with $1 \mathrm{~mL}$ of $80 \%$ methanol.

2.2. Total Phenolic Content. Total phenolic amount was measured by colorimetric assay with Folin \& Ciocalteu's reagent. Ten $\mu \mathrm{L}$ of appropriately diluted samples was mixed with $130 \mu \mathrm{L}$ of distilled water in a 96-well plate. Subsequently, $10 \mu \mathrm{L}$ of Folin \& Ciocalteu's was added. After $6 \mathrm{~min}$ reaction, $100 \mu \mathrm{L}$ of $7 \% \mathrm{Na}_{2} \mathrm{CO}_{3}$ solution was added. The absorbance of the mixture at $750 \mathrm{~nm}$ was measured after 90 min incubation in a microplate reader (ALLSHENG, Hangzhou, China). Total phenolic content in Sargassum species was expressed as mg gallic acid equivalents (GAE)/g dry weight.
2.3. Total Flavonoid Content. Total flavonoid contents were measure by the slightly modified Zhishen, Mengcheng method [8]. In a 96-well plate, $25 \mu \mathrm{L}$ of samples was mixed with $160 \mu \mathrm{L}$ of distilled water. $7.5 \mu \mathrm{L}$ of $5 \%$ sodium nitrite $\left(\mathrm{NaNO}_{2}\right)$ was added to this mixture. Five minutes later, $10 \%$ aluminum chloride $\left(\mathrm{AlCl}_{3}\right)$ was added. Another 6 minutes later, $50 \mu \mathrm{L}$ of $1 \mathrm{M}$ sodium hydroxide $(\mathrm{NaOH})$ was pipetted into designated wells. After mixing, the absorbance was measured against a blank at $510 \mathrm{~nm}$ with UV-spectrometer. Five milligrams of quercetin was dissolved in $80 \%$ methanol and then diluted to $25,50,100$, and $150 \mathrm{mg} / \mathrm{ml}$ as standards. Total flavonoid contents were expressed by mg quercetin equivalent (QE)/g dry weight (mg QE/g dw).

2.4. ABTS Radical Scavenging Assay. Antioxidant activity was measured using a group of assays. ABTS radical scavenging assay was slightly modified from the van den Berg method [9]. In brief, $1.0 \mathrm{mM}$ of $2,2^{\prime}$-azobis(2-amidinopropane) dihydrochloride (AAPH) was mixed with $2.5 \mathrm{mM}$ 2,2'-azino-bis(3-ethylbenzothiazoline-6-sulfonic acid) diammonium salt (ABTS) in $100 \mathrm{~mL}$ of phosphate-buffered solution (PBS). The mixture was heated in a water bath at $75^{\circ} \mathrm{C}$ for 30 min to generate ABTS radical. After the ABTS radical was produced, the radical solution was filtered through $0.45 \mu \mathrm{m}$ PVDF filter. The ABTS solution was diluted with PBS to make the absorbance at $734 \mathrm{~nm}$ between $0.650 \pm 0.020$. Four microliters of diluted Sargassum species samples was pipetted to $196 \mu \mathrm{L}$ of the ABTS radical solution. The mixture was incubated at $37^{\circ} \mathrm{C}$ for $10 \mathrm{~min}$. The decrease in absorbance at $734 \mathrm{~nm}$ was measured after $10 \mathrm{~min}$. The antioxidant capacity of Sargassum species was expressed as $\mathrm{mg}$ vitamin $\mathrm{C}$ equivalent (VCE)/g dry weight.

2.5. DPPH Radical Scavenging Assay. The DPPH assay was carried out according to Blois, M. S. method with a slight modification [10]. Briefly, $7.89 \mathrm{mg}$ of DPPH (2,2-diphenyl1-picrylhydrazyl) was dissolved into $80 \%(\mathrm{v} / \mathrm{v})$ aqueous methanol to make the concentration to $100 \mu \mathrm{M}$. Subsequently, the absorbance of DPPH radical solution was adjusted as $0.720 \pm 0.020$ with $80 \%$ methanol at $517 \mathrm{~nm}$. Five $\mu \mathrm{L}$ of diluted Sargassum species sample was mixed with $295 \mu \mathrm{L}$ of DPPH solution. The decreased absorbance of the sample and radical solvent mixture between sample extract and the radical solution was measured at $510 \mathrm{~nm}$ after reaction for $30 \mathrm{~min}$ in a dark room at room temperature. $80 \%$ aqueous methanol was used for the reference instead of samples, and vitamin $\mathrm{C}$ was used to make a standard curve. The DPPH radical scavenging capacity of Sargassum species was expressed as $\mathrm{mg} \mathrm{VCE} / \mathrm{g} \mathrm{dw}$.

2.6. Ferric Reducing Antioxidant Power (FRAP) Assay. Ferric reducing power of Sargassum species was measured using slightly modified Benzie and Strain's method [11]. FRAP reagent was prepared by mixing 10 volumes of acetate buffer $(300 \mathrm{mM}, \mathrm{pH} 3.6)$ with 1 volume of $10 \mathrm{mM}$ TPTZ (2,4,6-tri[2-pyridyl]-s-triazine) solution, 1 volume of $20 \mathrm{mM}$ of $\mathrm{FeCl} 3$ solution, and 1.2 volume of distilled water. The 
FRAP reagent was kept at $37^{\circ} \mathrm{C}$ until use. Samples were mixed with $250 \mu \mathrm{L}$ of FRAP reagent and incubated for $4 \mathrm{~min}$ at $37^{\circ} \mathrm{C}$. The absorbance was measured at $593 \mathrm{~nm}$. Ferrous sulphate $\left(\mathrm{FeSO}_{4}\right)$ was used to make a standard curve $(0.1 \mathrm{mM}$ to $1.0 \mathrm{mM})$. The reducing power was expressed as $\mathrm{mM} \mathrm{FeSO}_{4}$ equivalent/g dw.

2.7. Superoxide Radical Scavenging Assay. Superoxide radical scavenging activity was measured by the modified method of Zhang [12]. For measuring superoxide radical scavenging ability, $300 \mu \mathrm{M}$ nitroblue tetrazolium (NBT), $60 \mu \mathrm{M}$ of phenazine mepthosulphate (PMS), and $468 \mu \mathrm{M}$ of NADH were dissolved in $16 \mu \mathrm{M}$ Tris- $\mathrm{HCl}$ buffer ( $\mathrm{pH}$ 8.0). The sample was mixed with the same volume of NBT and NADH. Afterward, the same volume of PMS was injected for a reaction. In the reaction mixture that contained NADH and NBT, adding PMS initiates NADH oxidation to generate superoxide radicals to reduce NBT. After $5 \mathrm{~min}$ incubation at room temperature, the absorbance was measured at $560 \mathrm{~nm}$. Inhibition rate (\%) of superoxide radical scavenging assay was expressed as $\left(\mathrm{A}_{\text {blank }}-\mathrm{A}_{\text {sample }}\right) / \mathrm{A}_{\text {blank }} \times 100$.

2.8. Tyrosinase Inhibitor Activity. To measure tyrosinase inhibitor activity, first $110 \mu \mathrm{L}$ of $0.1 \mathrm{M}$ phosphate buffer saline (pH7.0), $10 \mu \mathrm{L}$ of the diluted sample, and $10 \mu \mathrm{L}$ of 1,000 units/ $\mathrm{ml}$ mushroom tyrosinase were injected in 96 well plates and incubated for $30 \mathrm{~min}$ at $37^{\circ} \mathrm{C}$. Additionally, $20 \mu \mathrm{L}$ of $1.5 \mathrm{mM}$ tyrosine was added to the incubated mixture. After incubating $15 \mathrm{~min}$ at $37^{\circ} \mathrm{C}$, the absorbance was measured at $490 \mathrm{~nm}$ using a spectrometer. Ten milligrams of kojic acid was dissolved in $0.1 \mathrm{M}$ PBS and then diluted to $62.5,125,250$, and $500 \mathrm{mg} / \mathrm{L}$ for standard. Tyrosinase inhibitor activity was expressed as $\mathrm{mg}$ kojic acid equivalent (KAE)/g dw. And, the tyrosinase inhibitory activity used by L-DOPA was measured in a microtube to add $170 \mu \mathrm{L}$ of $0.1 \mathrm{M}$ PBS (pH 7.0), $10 \mu \mathrm{L}$ of properly diluted samples, and $10 \mu \mathrm{L}$ of mushroom tyrosinase (1,000 units $/ \mathrm{ml})$ and mixed by using vortex. After transferring the mixture to 96 well plates, incubated for $6 \mathrm{~min}$ at $37^{\circ} \mathrm{C}, 10 \mu \mathrm{L}$ of $3 \mathrm{mML}$ DOPA was injected and incubated for $1 \mathrm{~min}$ at $37^{\circ} \mathrm{C}$. Absorbance was measured at $490 \mathrm{~nm}$. The standard for tyrosinase inhibitor activity with L-DOPA was calculated by using 31.12 , 62.50, and $125 \mathrm{mg} / \mathrm{L}$ kojic acid and expressed as $\mathrm{mg} \mathrm{KAE} / \mathrm{g} \mathrm{dw}$.

2.9. UPLC Chromatographic Analysis. Quantification of Sargahydroqunoic acid (SHQA) and sargachromanol (SCM) were conducted by Waters UPLC Aquity-PDA device. The column used was Acquity UPLC C18 $1.7 \mu \mathrm{M} \times 2.1 \times 50 \mathrm{~mm}$ column (Waters, Milford, MA, USA) for UPLC. And, column's temperature was $35^{\circ} \mathrm{C}$. The linear gradient of mobile phases between $0.1 \%$ formic acid in distilled water (Solvent A) and $0.1 \%$ formic acid in acetonitrile (Solvent B) was used as follows: $0 \mathrm{~min}(90: 10), 1 \mathrm{~min}(90: 10), 3 \mathrm{~min}(50: 50)$, $6 \min (30: 70), 11 \min (0: 100), 12 \min (0: 100), 15 \min (50:$ $50), 17 \mathrm{~min}(90: 10)$, and $19 \mathrm{~min}(90: 10)$. The flow rate was $0.400 \mathrm{ml} / \mathrm{min}$. And, scanning range is $210-600 \mathrm{~nm}$. Each compound was identified by retention time (RT). SHQA and SCM were detected at $270 \mathrm{~nm}$ absorbance. As a standard for quantification, purified SHQA and SCM were kindly provided by Dr. Heyung-Rak Kim's laboratory in Pukyong National University. The limit of detection and the limit of quantification were expressed $3{ }^{*} S_{\mathrm{a}} / b$ and $10{ }^{*} S_{\mathrm{a}} / b$, where $S_{\mathrm{a}}$ was the standard deviation of the reposed and $b$ was the slope of the standard curve, the standard deviation of $y$ residuals of regression line.

2.10. Statistical Analysis. All analyses were repeated three times. One-way ANOVA and Tukey's post hoc test were performed using Graphpad 7.0 (Graphpad Software, San Dieogo, USA). Significant differences between the results were analyzed using a Tukey post hoc test at a significance level of $P<0.05$.

\section{Results}

3.1. Total Phenolic and Total Flavonoid Contents. Phenolic compounds have a specific structure, hydroxy-substituted benzene ring, which can make them have strong antioxidant capacity. As shown in Table 1, the total phenolic content ranged from 20.57 to $88.97 \mathrm{mg}$ gallic acid equivalent of dry weight which was about 4 times different between the highest and lowest samples. S. miyabei Yendo $(88.97 \pm 4.34)$ and S.hemiphyllum $(83.22 \pm 1.72)$ exhibited the highest phenolic content, while $S$. confusum (20.57 \pm 1.00$)$, S. coreanum (23.82 \pm 0.88$)$, S. macrocarpum (26.32 \pm 1.72$)$, and $S$. micracanthum $(28.62 \pm 1.00)$ had the lowest.

Total flavonoid contents of Sargassum species are also listed in Table 1. The total flavonoid content ranged from 22.08 to $82.33 \mathrm{mg}$ quercetin equivalent of dry weight which was 3.7 times different between the highest and lowest samples. Same as phenolic content, the 2 species that showed the greatest amount of flavonoid were S. miyabei Yendo $(82.33 \pm 3.23)$ and $S$. hemiphyllum $(72.58 \pm 4.91)$, and S. coreanum $(22.08 \pm 0.65)$, S. macrocarpum $(22.08 \pm 1.32)$, S. confusum $(23.58 \pm 3.23)$, and $S$. micracanthum $(23.95 \pm 2.50)$ had the lowest flavonoid content.

3.2. Antioxidant Capacity. An array of assays was adopted to determine the antioxidant capacity of various species of Sargassum. As shown in Table 2, in the same with the total phenolic and flavonoid contents, antioxidant capacities showed a wide range. The strongest antioxidant activity was 8.8, 8.4, 2.2, and 36.8 times higher than the lowest activity, based on ABTS, DPPH, superoxide radical scavenging, and FRAP assays, respectively.

S. hemiphyllum and S. miyabei Yendo showed the highest ABTS and superoxide scavenging activities, whereas S. miyabei Yendo exhibited higher DPPH and FRAP activities than S. hemiphyllum.

The four species, S. coreanum, S. macrocarpum, S. confusum, and S. micracanthum, containing the lowest phenolic and flavonoid contents also showed the lowest ABTS, $\mathrm{DPPH}$, and FRAP activities. The range of superoxide radical scavenging activity was less wide compared to the other three activities. There were no significant differences among 10 out of 
TABLE 1: Total phenolic and total flavonoid contents of Sargassum species.

\begin{tabular}{lcc}
\hline & Total phenolic content $(\mathrm{mg} \mathrm{GAE} / \mathrm{g} \mathrm{dw})$ & Total flavonoid content (mg QE/g dw) \\
\hline S. confusum & $20.57 \pm 1.00^{\mathrm{d}}$ & $23.58 \pm 3.23^{\mathrm{d}}$ \\
S. coreanum & $23.82 \pm 0.88^{\mathrm{d}}$ & $22.08 \pm 0.65^{\mathrm{d}}$ \\
S. filicinum & $56.21 \pm 1.00^{\mathrm{b}}$ & $65.38 \pm 7.77^{\mathrm{b}}$ \\
S. hemiphyllum & $83.22 \pm 1.72^{\mathrm{a}}$ & $72.58 \pm 4.91^{\mathrm{ab}}$ \\
S. horneri & $36.09 \pm 1.00^{\mathrm{c}}$ & $36.58 \pm 2.63^{\mathrm{c}}$ \\
S. macrocarpum & $26.32 \pm 1.72^{\mathrm{d}}$ & $22.08 \pm 1.32^{\mathrm{d}}$ \\
S. micracanthum & $28.62 \pm 1.00^{\mathrm{d}}$ & $23.95 \pm 2.50^{\mathrm{d}}$ \\
S. miyabei Yendo & $88.97 \pm 4.34^{\mathrm{a}}$ & $82.33 \pm 3.24^{\mathrm{a}}$ \\
S. serratifolium & $55.63 \pm 6.22^{\mathrm{b}}$ & $46.83 \pm 2.48^{\mathrm{c}}$ \\
S. siliquastrum & $40.11 \pm 1.72^{\mathrm{c}}$ & $32.95 \pm 2.02^{\mathrm{cd}}$ \\
S. yendoi & $42.41 \pm 1.00^{\mathrm{c}}$ & $40.70 \pm 4.20^{\mathrm{c}}$ \\
\hline
\end{tabular}

TPC and TFC contents were expressed as mg gallic acid equivalent (GAE)/g dry weight and mg quercetin equivalent (QE)/g dry weight. Data are presented as mean \pm SD. Groups that do not share a common letter indicate a significant difference $(P<0.05)$.

TABle 2: Antioxidant capacity of Sargassum species.

\begin{tabular}{|c|c|c|c|c|}
\hline & ABTS (mg VCE/g dw) & DPPH (mg VCE/g dw) & Superoxide radical (\%) & FRAP $\left(\mathrm{mM} \mathrm{Fe}^{2+}\right.$ equivalent/g dw) \\
\hline S. confusum & $25.65 \pm 1.22^{\mathrm{d}}$ & $38.56 \pm 4.82^{\mathrm{d}}$ & $36.71 \pm 0.77^{b}$ & $0.026 \pm 0.003^{\mathrm{de}}$ \\
\hline S. coreanum & $32.59 \pm 0.46^{\mathrm{d}}$ & $39.96 \pm 3.38^{\mathrm{cd}}$ & $23.11 \pm 2.30^{b c}$ & $0.092 \pm 0.008^{\mathrm{d}}$ \\
\hline S. filicinum & $140.05 \pm 4.69^{\mathrm{b}}$ & $78.21 \pm 0.61^{\mathrm{c}}$ & $40.39 \pm 1.80^{\mathrm{b}}$ & $0.158 \pm 0.011^{\mathrm{cd}}$ \\
\hline S. hemiphyllum & $205.65 \pm 7.17^{\mathrm{a}}$ & $137.86 \pm 5.40^{\mathrm{b}}$ & $51.94 \pm 3.07^{\mathrm{a}}$ & $0.645 \pm 0.065^{\mathrm{b}}$ \\
\hline S. horneri & $74.19 \pm 3.70^{c}$ & $61.02 \pm 1.61^{\mathrm{c}}$ & $30.98 \pm 1.11^{b c}$ & $0.135 \pm 0.020^{\mathrm{d}}$ \\
\hline S. macrocarpum & $32.85 \pm 1.22^{\mathrm{d}}$ & $27.68 \pm 0.61^{\mathrm{d}}$ & $32.21 \pm 3.54^{\mathrm{bc}}$ & $0.068 \pm 0.006^{\mathrm{d}}$ \\
\hline S. micracanthum & $23.25 \pm 0.46^{\mathrm{d}}$ & $23.12 \pm 2.43^{\mathrm{d}}$ & $34.76 \pm 2.26^{\mathrm{b}}$ & $0.038 \pm 0.020^{\mathrm{d}}$ \\
\hline S. miyabei Yendo & $186.19 \pm 23.15^{\mathrm{a}}$ & $193.65 \pm 23.19^{\mathrm{a}}$ & $43.66 \pm 3.38^{\mathrm{ab}}$ & $0.956 \pm 0.094^{\mathrm{a}}$ \\
\hline S. serratifolium & $99.52 \pm 2.88^{\mathrm{c}}$ & $75.40 \pm 1.82^{\mathrm{c}}$ & $34.76 \pm 3.38^{\mathrm{b}}$ & $0.286 \pm 0.020^{\mathrm{c}}$ \\
\hline S. siliquastrum & $76.32 \pm 2.12^{c}$ & $66.28 \pm 4.86^{\mathrm{c}}$ & $40.39 \pm 0.71^{\mathrm{ab}}$ & $0.195 \pm 0.018^{\mathrm{c}}$ \\
\hline S. yendoi & $89.39 \pm 4.41^{\mathrm{c}}$ & $59.61 \pm 2.79^{\mathrm{cd}}$ & $38.04 \pm 5.07^{b}$ & $0.236 \pm 0.043^{c}$ \\
\hline
\end{tabular}

TAC: total antioxidant capacity; ABTS: ABTS radical scavenging capacity; DPPH: DPPH radical scavenging capacity; superoxide radical: superoxide radical scavenging capacity; FRAP: ferric-reducing antioxidant power. Data are presented as mean \pm SD. Groups tht do not share a common letter indicate a significant difference $(P<0.05)$.

the 11 Sargassum species, except for S. hemiphyllum, which showed the highest scavenging activity.

3.3. Measurement of Tyrosinase Inhibitor Activity with L-Tyrosine and L-DOPA. Tyrosinase is an enzyme responsible for the synthesis of melanin, the pigment responsible for skin color. Presently several natural or synthetic compounds have been used to inhibit tyrosinase and have also been used by the cosmetic industry as a skin whitening and anti-wrinkling agent [13]. Tyrosinase inhibition activity assay results are shown in $\mathrm{Ta}$ ble 3. It was expressed as mg kojic acid equivalent per gram of dry weight. When tyrosine was used as substrate, $S$. yendoi $(126.30 \pm 0.50)$ showed the highest anti-tyrosinase activity, followed by $S$. filicinum $(117.63 \pm 1.61)$, S. hemiphyllum $(88.60 \pm 3.61)$, and $S$. miyabei Yendo $(70.47 \pm 2.57)$. When L-DOPA was used as a substrate, S. yendoi $(52.65 \pm 0.14)$, S. filicinum $(50.07 \pm 1.23)$, and S. hemiphyllum $(47.330 \pm 1.61)$ showed the highest tyrosinase inhibition ability.

3.4. Correlation between Antioxidant Capacity and Anti-Tyrosinase Activity. To determine the association between total phenolic contents, total flavonoid contents, and antioxidant ability in 11 species of Sargassum, Pearson correlation
TABle 3: Tyrosinase inhibition activity of Sargassums.

\begin{tabular}{lcc}
\hline & $\begin{array}{c}\text { L-tyrosine } \\
\text { (mg KAE/g dw) }\end{array}$ & $\begin{array}{c}\text { L-DOPA } \\
\text { (mg KAE/g dw) }\end{array}$ \\
\hline S. confusum & $15.63 \pm 1.76^{\mathrm{g}}$ & $19.65 \pm 3.11^{\mathrm{b}}$ \\
S. coreanum & $18.97 \pm 4.31^{\mathrm{g}}$ & $26.23 \pm 11.13^{\mathrm{b}}$ \\
S. filicinum & $117.63 \pm 1.61^{\mathrm{b}}$ & $50.07 \pm 1.23^{\mathrm{a}}$ \\
S. hemiphyllum & $88.6 \pm 3.61^{\mathrm{c}}$ & $47.3 \pm 1.61^{\mathrm{a}}$ \\
S. horneri & $43.47 \pm 1.44^{\mathrm{e}}$ & $30.32 \pm 1.15^{\mathrm{b}}$ \\
S. macrocarpum & $14.63 \pm 2.36^{\mathrm{g}}$ & $19.57 \pm 0.88^{\mathrm{b}}$ \\
S. micracanthum & $13.30 \pm 0.50^{\mathrm{g}}$ & $19.98 \pm 0.75^{\mathrm{b}}$ \\
S. miyabei Yendo & $70.47 \pm 2.57^{\mathrm{d}}$ & $26.07 \pm 2.92^{\mathrm{b}}$ \\
S. serratifolium & $50.47 \pm 4.16^{\mathrm{e}}$ & $29.73 \pm 7.81^{\mathrm{b}}$ \\
S. siliquastrum & $41.97 \pm 0.76^{\mathrm{f}}$ & $24.32 \pm 1.53^{\mathrm{b}}$ \\
S. yendoi & $126.3 \pm 0.50^{\mathrm{a}}$ & $52.65 \pm 0.14^{\mathrm{a}}$ \\
\hline
\end{tabular}

L-tyrosine and L-DOPA were used to measure the tyrosinase inhibitor ability. Tyrosinase inhibition abilities were expressed as mg kojic acid equivalent $(\mathrm{KAE}) / \mathrm{g} \mathrm{dw}$. Different letters indicate significant difference $(P<0.05)$.

analysis was conducted. In Table 4, the results showed that the total phenolic contents, total flavonoid contents, and antioxidant ability were significantly positively correlated. Through this, it was confirmed that various methods of measuring antioxidant capacity could be used to measure the antioxidant capacity of Sargassum. Besides, the total phenolic content and flavonoid content showed a significant 
TABLE 4: Correlation between TPC, TFC, and four TAC values.

\begin{tabular}{lcccccc}
\hline & TPC & TFC & ABTS & DPPH & FRAP & Superoxide scavenging \\
\hline TPC & 1 & $0.97^{* *}$ & $0.97^{* *}$ & $0.95^{* *}$ & $0.93^{* *}$ & $0.77^{* *}$ \\
TFC & - & 1 & $0.98^{-* *}$ & $0.92^{* *}$ & $0.87^{* *}$ & $0.75^{-* *}$ \\
ABTS & - & - & 1 & $0.91^{* *}$ & $0.87^{* *}$ & $0.80^{* *}$ \\
DPPH & - & - & - & 1 & $0.98^{* *}$ & $0.69^{*}$ \\
FRAP & - & - & - & - & 1 & $0.67^{*}$ \\
Superoxide scavenging & - & - & - & - & - & 1 \\
\hline
\end{tabular}

TPA, TFA, TAC stand for total phenolic amount, total flavonoid amount, and total antioxidant capacity, respectively. DPPH radical scavenging capacity, ABTS radical scavenging capacity, superoxide radical scavenging capacity, and ferric-reducing antioxidant power were measured for the analysis of TAC. $\left({ }^{*} \mathrm{P}<0.05 ;{ }^{* *} \mathrm{P}<0.01\right)$.

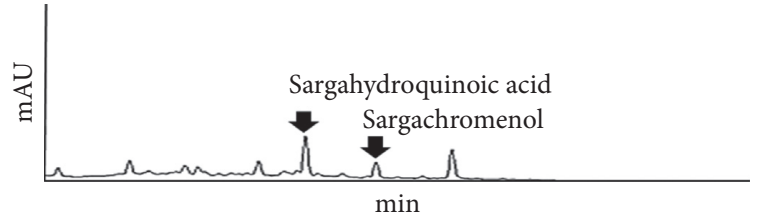

(a)

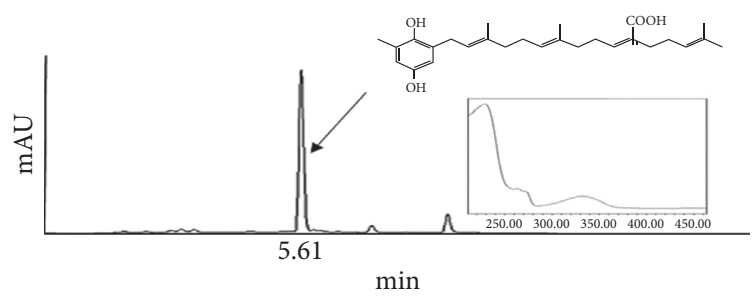

(c)

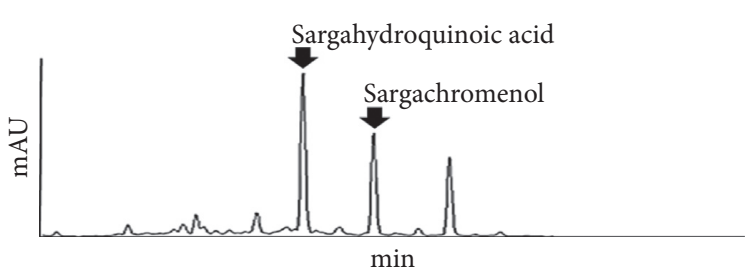

(b)

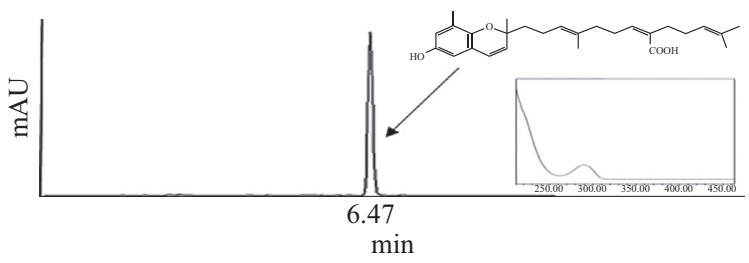

(d)

Figure 1: UPLC Chromatograms of (a) S. serratifolium, (b) S. miyabei Yendo, (c) sargahydroquinoic acid, and (d) sargachromenol standards.

positive correlation, indicating that flavonoid contributes largely to the polyphenol content in Sargassum.

3.5. Quantification of SHQA and SCM. As shown in Figure 1, SCM and SHQA were measured through UPLC in 11 Sargassum species. Retention time (RT) of SHQA and SCM purified standards was detected at 5.60 and $6.68 \mathrm{~min}$, respectively. The limit of detection (LOD) and the limit of quantification (LOQ) of SCM were $0.014 \mathrm{mg} / \mathrm{L}$ and $0.045 \mathrm{mg} / \mathrm{L}$, and LOD and LOQ of SHQA were $0.28 \mathrm{mg} / \mathrm{L}$ and $0.933 \mathrm{mg} / \mathrm{L}$. Among the 11 Sargassum species extracts, SHQA and SCM were detected in S. miyabei Yendo and S. serratifolium. In the extract of S. miyabei Yendo, SHQA was quantified as $118.44 \pm 0.87 \mathrm{mg} / \mathrm{g}$ and SCM as $17.82 \pm 0.10 \mathrm{mg} / \mathrm{g}$. Also, S. serratifolium extract contained $17.75 \pm 0.12 \mathrm{mg} / \mathrm{g}$ SHQA and $1.05 \pm 0.01 \mathrm{mg} / \mathrm{g}$ SCM.

\section{Discussion}

The Republic of Korea, as a major producer of maricultured seaweed, has experience in artificial production and cultivation of Sargassum species [14, 15]. In an effort to identify the Sargassum species growing off the Korean coast and develop the nutrient intensive Sargassum species for cultivation, all the accessible Sargassum species off the Korean coast were collected from MBRIS and their polyphenol contents were analyzed.

A regular intake of phenolic compounds exhibits a potential for the prevention or reduction in the risk of degenerative disease associated with oxidative stress, such as cardiovascular disease, diabetes, and obesity [16, 17]. Among the phenolic compounds, flavonoids are the largest group that has received major attention in research. TPC and TFC from several Sargassum species had been tested in other studies. However, due to the differences in extraction methods and different equivalents used, the results from different studies were incomparable. For example, TPC of methanol extracts from $S$. horneri was $0.51 \pm 0.02 \mathrm{mg} \mathrm{GAE} / \mathrm{g}$ in Yin and Woo's research, while the analysis of Generalic Mekinic showed $9.90 \mathrm{mg}$ pyrocatechol equivalent/g in methanol extract of $S$. horneri. [18-21]. In our study, TPC and TFC of a variety of Sargassum species were analyzed and the highest concentrations were discovered in S. miyabei Yendo and S. hemiphyllum. Furthermore, by using the same equivalent, we found that our results suggest that flavonoids were the major phenolic contents in Sargassum species, which is corroborated by the strong Pearson correlation $(R=0.97)$ between TPC and TFC.

The total antioxidant capacities were analyzed by ABTS, DPPH, FRAP, and superoxide radical scavenging assays, 
which are widely used approaches to determine the antioxidant capacities of plant extracts. They gave comparable results in the present study with minor differences. Overall, S. hemiphyllum and S. miyabei Yendo, the two species containing the highest TPC and TFC, displayed the strongest antioxidant capacity. In addition to our findings, the strong antioxidant capacities, anti-inflammatory activities, and antihyperglycemic activities of S. hemiphyllum and S. miyabei Yendo have been previously reported [22], $[23,24],[25,26]$.

Tyrosinase is critical in the melanogenesis and enzymatic browning. Its inhibitors attract great attention in the cosmetic industry as depigmentation agents and in the food industry as antibrowning compounds. Phenolic compounds can inhibit tyrosinase competitively by mimicking the substrate of tyrosinase [7]. Our research indicated that S. fillicinum and S. yendoi, which contained moderate amounts of phenolics and flavonoids showed the strongest inhibition against tyrosinase. This suggests that the composition rather than the concentration of phenolics have a bigger influence on tyrosinase. In addition, nonphenolic compounds may also exert anti-tyrosinase activity. For example, astaxanthin and fucoxanthin, two carotenoids that occurring naturally in brown algae, had shown anti-tyrosinase activities $[27,28]$. Further studies are needed to identify the bioactive compounds in the extracts that exert strong anti-tyrosinase activity.

SCM and SHQA are known bioactive components in Sargassum species. The structure of SCM shows that SCM is a natural homologue of $\delta$-tocotrienol with an additional carboxyl group in polyphenyl tail [29]. SHQA and SCM are two bioactive components isolated from various Sargassum species that have drawn growing attention. These two meroterpenoid compounds have shown various biological benefits. For example, SCM and SHQA extracted from S. serratifolium showed anti-inflammatory, anti-hyperpigmentation, and anti-obesity activities in vivo and in vitro studies, [30], [31]. Our research showed that only two species exhibited detectable amounts of SCM and SHQA among 11 Sargassum species. Further studies are needed to have a more comprehensive investigation of the health benefits of Sargassum species and maximize the commercial usage of these species.

\section{Data Availability}

The data that support the findings of this study are available from the corresponding author upon reasonable request.

\section{Conflicts of Interest}

The authors declare that they have no conflicts of interest.

\section{Acknowledgments}

This research was a part of the project titled "Future fisheries food research center", funded by the Ministry of Oceans and Fisheries, Korea, and was also supported by the National Research Foundation of Korea (NRF) grant funded by the
Korea government (Ministry of Science, ICT \& Future Planning (MSIP)) (no. CD20200160).

\section{References}

[1] T. Chopin and A. G. J. Tacon, "Importance of seaweeds and extractive species in global aquaculture production," Reviews in Fisheries Science \& Aquaculture, pp. 1-10, 2020.

[2] M. Guiry, AlgaeBase. World-wide Electronic Publication, National University of Ireland, Galway, Ireland, 2010, http:// www. algaebase. org/.

[3] S. Yende, B. Chaugule, and U. Harle, "Therapeutic potential and health benefits ofSargassumspecies," Pharmacognosy Reviews, vol. 8, no. 15, pp. 1-7, 2014.

[4] K. Apel and H. Hirt, "Reactive oxygen species: metabolism, oxidative stress, and signal transduction," Annual Review of Plant Biology, vol. 55, no. 1, pp. 373-399, 2004.

[5] Y. Wang, M. Yang, S.-G. Lee, C. G. Davis, S. I. Koo, and O. K. Chun, "Dietary total antioxidant capacity is associated with diet and plasma antioxidant status in healthy young adults," Journal of the Academy of Nutrition and Dietetics, vol. 112, no. 10, pp. 1626-1635, 2012.

[6] N. Munir, N. Sharif, S. Naz, and F. Manzoor, "Algae: a potent antioxidant source," Sky Journal of Microbiology Research, vol. 1, no. 3, pp. 22-31, 2013.

[7] S. Zolghadri, A. Bahrami, M. T. Hassan Khan et al., "A comprehensive review on tyrosinase inhibitors," Journal of Enzyme Inhibition and Medicinal Chemistry, vol. 34, no. 1, pp. 279-309, 2019.

[8] T. M. Jia Zhishen and J.. Wu, "The determination of flavonoid contents in mulberry and their scavenging effects on superoxide radicals," Food Chemistry, vol. 64, no. 4, pp. 555-559, 1998.

[9] R. Van Den Berg, G. R. M. M. Haenen, H. Van Den Berg, and A. Bast, "Applicability of an improved Trolox equivalent antioxidant capacity (TEAC) assay for evaluation of antioxidant capacity measurements of mixtures," Food Chemistry, vol. 66, no. 4, pp. 511-517, 1999.

[10] W. Brand-Williams and C. Berset, "Use of a free radical method to evalute antioxidant activity," Food Sicence and Technology, vol. 28, no. 1, pp. 25-30, 1994.

[11] I. F. F. Benzie, J. J. S. Benzie, and J. J. Strain, "The ferric reducing ability of plasma (FRAP) as a measure of "antioxidant power": the FRAP assay," Analytical Biochemistry, vol. 239, no. 1, pp. 70-76, 1996.

[12] Q.-F. Zhang, Z.-R. Zhang, and H.-Y. Cheung, "Antioxidant activity of Rhizoma Smilacis Glabrae extracts and its key constituent-astilbin," Food Chemistry, vol. 115, no. 1, pp. 297-303, 2009.

[13] E. J. K. Mi Ji Jeon, G. T. Kim, Ga Y. Kim, and S. J. Lee, "In Cheol Jung,Sang-young Kim, and young min Kim whitening effect and skin regeneration effect of red sea cucumber extract," Journal of Life Science, vol. 28, no. 6, pp. 681-687, 2018.

[14] E. K. Hwang, C. S. Park, and J. M. Baek, "Artificial Seed production and cultivation of the edible brown alga, Sargassum Fulvellum (turner) c. agardh: developing a new species for seaweed cultivation in korea," Journal of Applied Phycology, vol. 18, 2006.

[15] S. Redmond, J. K. Kim, C. Yarish, M. Pietrak, and I. Bricknell, Culture of Sargassum in Korea: Techniques and Potential for Culture in the US, Maine Sea Grant, Orono, ME, USA, 2014.

[16] C. W. I. Haminiuk, G. M. Maciel, M. S. V. Plata-Oviedo, and R. M. Peralta, "Phenolic compounds in fruits-an overview," 
International Journal of Food Science \& Technology, vol. 47, no. 10, pp. 2023-2044, 2012.

[17] C. Rodríguez-Pérez, A. Segura-Carretero, and M. Del Mar Contreras, "Phenolic compounds as natural and multifunctional anti-obesity agents: a review," Critical Reviews in Food Science and Nutrition, vol. 59, no. 8, pp. 1212-1229, 2019.

[18] T. T. Dang, M. C. Bowyer, I. A. Van Altena, and C. J. Scarlett, "Comparison of chemical profile and antioxidant properties of the brown algae," International Journal of Food Science \& Technology, vol. 53, no. 1, pp. 174-181, 2018.

[19] I. Generalic Mekinic, D. Skroza, V. Simat, I. Hamed, M. Cagalj, and Z. Popovic Perkovic, "Phenolic content of Brown algae (pheophyceae) species: extraction, identification, and quantification," Biomolecules, vol. 9, no. 6, 2019.

[20] H. Nagappan, P. P. Pee, S. H. Y. Kee et al., "Malaysian brown seaweeds Sargassum siliquosum and Sargassum polycystum: low density lipoprotein (LDL) oxidation, angiotensin converting enzyme (ACE), $\alpha$-amylase, and $\alpha$-glucosidase inhibition activities," Food Research International, vol. 99, no. 2, pp. 950-958, 2017.

[21] S. Yin, H.-C. Woo, J.-H. Choi, Y.-B. Park, and B.-S. Chun, "Measurement of antioxidant activities and phenolic and flavonoid contents of the brown seaweed Sargassum horneri: comparison of supercritical CO 2 and various solvent extractions," Fisheries and Aquatic Sciences, vol. 18, no. 2, pp. 123-130, 2015.

[22] P. A. Hwang, S. Y. Chien, Y. L. Chan et al., "Inhibition of Lipopolysaccharide (LPS)-induced inflammatory responses by Sargassum hemiphyllum sulfated polysaccharide extract in RAW 264.7 macrophage cells," Journal of Agricultural and Food Chemistry Journal, vol. 59, no. 5, pp. 2062-2068, 2011.

[23] P.-A. Hwang, Y.-L. Hung, Y.-K. Tsai, S.-Y. Chien, and Z.-L. Kong, "The brown seaweed Sargassum hemiphyllum exhibits $\alpha$-amylase and $\alpha$-glucosidase inhibitory activity and enhances insulin release in vitro," Cytotechnology, vol. 67, no. 4, pp. 653-660, 2015.

[24] H.-J. Na, P.-D. Moon, S.-G. Ko et al., "Sargassum hemiphyllum inhibits atopic allergic reaction via the regulation of inflammatory mediators," Journal of Pharmacological Sciences, vol. 97, no. 2, pp. 219-226, 2005.

[25] M.-J. Kim, N.-Y. Bae, K.-B.-W.-R. Kim et al., “Anti-inflammatory activity of ethanol extract of Sargassum miyabei yendo via inhibition of NF-B and MAPK activation," Microbiology and Biotechnology Letters, vol. 44, no. 4, pp. 442-451, 2016.

[26] M.-J. Yim, J. M. Lee, H.-S. Kim et al., "Inhibitory effects of a Sargassum miyabei yendo on cutibacterium acnes-induced skin inflammation," Nutrients, vol. 12, no. 9, p. 2620, 2020.

[27] A. R. Rao, H. N. Sindhuja, S. M. Dharmesh, K. U. Sankar, R. Sarada, and G. A. Ravishankar, "Effective inhibition of skin cancer, tyrosinase, and antioxidative properties by astaxanthin and astaxanthin esters from the green alga Haematococcus pluvialis," Journal of Agricultural and Food Chemistry, vol. 61, no. 16, pp. 3842-3851, 2013.

[28] H. Shimoda, J. Tanaka, S.-J. Shan, and T. Maoka, "Antipigmentary activity of fucoxanthin and its influence on skin mRNA expression of melanogenic molecules," Journal of Pharmacy and Pharmacology, vol. 62, no. 9, pp. 1137-1145, 2010.

[29] M. Birringer, K. Siems, A. Maxones, J. Frank, and S. Lorkowski, "Natural 6-hydroxy-chromanols and -chromenols: structural diversity, biosynthetic pathways and health implications," RSC Advances, vol. 8, no. 9, pp. 4803-4841, 2018.
[30] M. S. Azam, E.-J. Joung, J. Choi, and H.-R. Kim, "Ethanolic extract from Sargassum serratifolium attenuates hyperpigmentation through CREB/ERK signaling pathways in $\alpha$-MSH-stimulated B16F10 melanoma cells," Journal of Applied Phycology, vol. 29, no. 4, pp. 2089-2096, 2017.

[31] S.-N. Kim, H. Y. Choi, W. Lee, G. M. Park, W. S. Shin, and Y. K. Kim, "Sargaquinoic acid and sargahydroquinoic acid from Sargassum yezoense stimulate adipocyte differentiation through PPAR $\alpha / \gamma$ activation in 3T3-L1 cells," FEBS Letters, vol. 582, no. 23-24, pp. 3465-3472, 2008. 